\title{
Color effects associated with the 1999 microlensing brightness peaks in gravitationally lensed quasar Q2237+0305
}

\author{
V. G. Vakulik ${ }^{1}$, R. E. Schild ${ }^{2}$, V. N. Dudinov ${ }^{1}$, A. A. Minakov ${ }^{3}$, S. N. Nuritdinov ${ }^{4}$, V. S. Tsvetkova ${ }^{3}$, \\ A. P. Zheleznyak ${ }^{1}$, V. V. Konichek ${ }^{1}$, I. Ye. Sinelnikov ${ }^{1}$, O. A. Burkhonov ${ }^{4}$, \\ B. P. Artamonov ${ }^{5}$, and V. V. Bruevich ${ }^{5}$ \\ ${ }^{1}$ Institute of Astronomy of Kharkov National University, Sumskaya 35, 61022 Kharkov, Ukraine \\ 2 Center for Astrophysics, 60 Garden Street, Cambridge, MA 02138, USA \\ e-mail: rschild@cfa.harvard.edu \\ ${ }^{3}$ Institute of Radio Astronomy of Nat. Ac. Sci. of Ukraine, Chervonoznamennaya 4, 61002 Kharkov, Ukraine \\ e-mail: minakov@ira.kharkov.ua \\ ${ }^{4}$ Ulugh Beg Astronomical Institute of Ac. Sci. of Uzbekistan, Astronomicheskaya 33, 700052, Tashkent, \\ Republic of Uzbekistan \\ e-mail: nurit@astrin.uzsci.net \\ 5 Sternberg Astronomical Institute, Universitetski Av. 13, 119899 Moscow, Russia \\ e-mail: artamon@sai.msu.ru
}

Received 23 July 2003 / Accepted 9 February 2004

\begin{abstract}
We present photometry of the Q2237+0305 gravitational lens system in VRI spectral bands with the 1.5-m telescope of the high-altitude Maidanak observatory in 1995-2000. The time interval includes the epoch of the dramatic brightness peaks discovered previously in the A and C image components (Wozniak et al. 2000a,b). By good luck three nights of observation in 1999 were almost at the time of the strong brightness peak of image $\mathrm{C}$, and approximately in the middle of the ascending slope of the brightness peak of image A. Having reached its brightness maximum at the very end of June 1999, the C component had changed its $(V-I)$ color from $0.3^{\mathrm{m}}$ to $0.12^{\mathrm{m}}$ since August 1998 , and from $0.56^{\mathrm{m}}$ to $0.12^{\mathrm{m}}$ since August 1997. It was the bluest component in the system in 1998 and 1999, but by October 2000 that was no longer the case. We do not know the color of the A component exactly at its brightness peak, but we do know that it became $0.47^{\mathrm{m}}$ brighter in $R$ and $0.15^{\mathrm{m}}$ bluer in $(V-R)$ between August 1998 and August 2000, about three months before the peak. More intensive monitoring of Q2237+0305 in July-October 2000, made on a nearly daily basis, did not reveal rapid (night-to-night and intranight) brightness variations of the components during this time period, exceeding the photometry error bars. Rather slow changes of magnitudes of the components were observed, in particular, nearly synchronous $0.08^{\mathrm{m}}$ fading of $\mathrm{B}$ and $\mathrm{C}$ components, and $0.05^{\mathrm{m}}$ brightening of $\mathrm{D}$ in the $R$ band during July 23-October 7, 2000, while the B component had become the faintest in all filters by the end of this time period. The behavior of the colors of the components was analyzed on the basis of all our VRI observations, made in 1995-2000 on Maidanak. A qualitative tendency of the components to become bluer as their brightness increases, noted in our previous works, was confirmed quantitatively. A correlation between the color variations and variations of magnitudes of the components is demonstrated to be significant and reaches 0.75 for $\Delta(V-I)$ vs. $\Delta R$, with a regression line slope of $0.33 \pm 0.08$ for these quantities. A plot of $(V-I)$ vs. $(V-R)$ shows the components settled in a cluster, stretchng along a line with a slope of $1.31 \pm 0.14$. Both slopes are noticeably less steep than those expected if a standard galactic interstellar reddening law were responsible for the differences between the colors of images and their variations over time. We attribute the brightness and color changes to microlensing of the quasar's structure, which we conclude to be more compact at shorter wavelengths, as predicted by most quasar models featuring an energizing central source.
\end{abstract}

Key words. cosmology: gravitational lensing - galaxies: quasars: individual: QSO 2237+0305 - methods: observational techniques: image processing

Send offprint requests to: V.Vakulik, e-mail: vakulik@astron.kharkov.ua

* Tables 4-7 are only available in electronic form at the CDS via anonymous ftp to cdsarc.u-strasbg.fr $(130.79 .128 .5)$ or via http://cdsweb.u-strasbg/cgi-bin/qcat?]/A+A/420/447

\section{Introduction}

The Q2237+0305 gravitational lens (the Einstein Cross) is one of the most impressive manifestations of the gravitational lensing phenomenon - four images of the same high-redshift quasar $(z=1.695)$ are arranged almost symmetrically around the lensing galaxy nucleus $(z=0.039)$ within a circle of 
approximately $2^{\prime \prime}$ diameter. The Q2237+0305 system is an excellent target for studying microlensing events, because the light beams, corresponding to the 4 lensed quasar's images, pass through the inner, heavily populated part of the lensing galaxy, and thus have a high probability to intersect a significant mass of microlensing stars as they pass through the inner disc (Kayser \& Refsdal 1989).

The system has been intensively examined since 1987, when the first published measurements of magnitudes of the individual lensed quasar components in $g, r$ and $i$ Gunn filters were made by Yee (1988). The first attempt to build the light curves of the four quasar components was made in 1991 by Corrigan et al. (1991). They brought together all the available Q2237 images of sufficient quality, taken with different telescopes and in a variety of passbands, - Mould $B, V$ and $R$ and Gunn $g, r$ and $i$, - and reprocessed with a single algorithm. Having used the multicolor photometry data for 33 normal stars, whose $(B-V)$ colors ranged from -0.3 to 1.5 , they calculated the relevant color equations, which allowed them to reduce all the observations to a single passband. Their $r$ Gunn and $B$ light curves cover the time period from September 1986 up to December 1989, and include the first microlensing event observed in August 1988 by Irwin et al. (1989).

A further attempt to use all the available observational data for Q2237+0305 was made in 1994 by constructing "differential" light curves, which were argued to be free from the effects of different spectral bands, technique of zero-pointing, and the quasar intrinsic brightness variations (Houde et al. 1994). In addition to the data of 1986-89, contained in Corrigan et al. (1991), other results were used, taken in 1990 and 1991 by Crane et al. (1991), Racine (1992), Rix et al. (1992), and Houde et al. (1994).

The first program of regular photometric monitoring of Q2237+0305 was started in 1990 at the Nordic Optical Telescope, (Østensen et al. 1996). A large number of measurements of the four quasar components in $V, R$ and $I$ spectral bands during five years were obtained, which, with the use of Corrigan et al. (1991) zero-pointing, permitted the construction of the historic light curves, covering 9 years of observations.

No regular multi-filter monitoring of Q2237+0305 is reported between 1996 and 1999, excepting our results of VRI photometry for three nights on 17-19 September 1995, (Vakulik et al. 1997), and the similar results by Burud et al. (1998), obtained with the Nordic Optical Telescope for a nearby epoch, 10-11 October 1995. No night-to-night or intranight brightness variations of the four components was found for these time periods, while a noticeable change in the component B color as compared to the observations by Yee in 1987 (1988) was reported in both studies. Short time-scale monitoring with the CFHT in June 14-16, 1992 should be mentioned here (Cumming \& De Robertis 1995); it also did not reveal any photometric variations in $R$ and $I$ bands during a three-day period.

In 1999 and 2000, the results of VRI photometry in 1997 and 1998 with the Maidanak 1.5-m telescope were published (Bliokh et al. 1999; Dudinov et al. 2000a). Recently, the superb results of a detailed long-term monitoring, obtained within the OGLE program from 4 August 1997 to 5 November 2000 have become publicly accessible, and have been partly presented in two papers of Wozniak et al. (2000a,b). In 2002, the results of monitoring Q2237+0305 by the GLITP (Gravitational Lenses International Time Project) collaboration appeared, which cover the 4-month period October 1999-February 2000 (Alcalde et al. 2002). The most recent publication of the results of low-resolution observations with the 3.5-m telescope at the Apache Point Observatory should also be mentioned here, (Schmidt et al. 2002); these gave the Gunn $r$ lightcurves of the A and B components for 73 dates between July 1995 and January 1998. In spite of a rather low photometric accuracy, an error bar of $0.1^{\mathrm{m}}$ to $0.2^{\mathrm{m}}$ is reported - the data are of value first of all because they include the brightness peak of the A component in 1996. As far as we know, no more data about this event have ever been published, though they were reported in private communication, (e.g. Østensen).

The brightness records taken in broad-band filters are a good starting point for theorists to estimate the size of the quasar radiating region in the visual (ultraviolet rest frame) continuum, and to determine the range of microlens masses responsible for the observed brightness variations. Both the statistical analysis of long-term monitoring data, and simulation of the isolated microlensing peaks have been applied to calculate these values (Nadeau et al. 1991; Lewis \& Irwin 1996; Refsdal \& Stabell 1993; Webster et al. 1991; Wyithe et al. 2000a,b, 2002; Yonehara 2000). These have given the estimates of the quasar dimension $-10^{15} \mathrm{~cm}$ to $10^{16} \mathrm{~cm}$ in the optical continuum, and a great variety of microlens masses estimates ranging from $0.0006 M_{\odot}<M<0.006 M_{\odot}$ (Nadeau et al. 1991), to $0.1 M_{\odot}$ (Wambsganss 1991), all indicating however, that microlensing events in the system are mostly caused by subsolar-mass objects.

These available models give rise to a problem which probably indicates a breakdown of the simple assumption that the luminous quasar is a uniformly bright accretion disc. The dilemma is that the observed events like the brightness peak in image C in July 1999 and the brightness peak in A in November 1999 occur on such short time scales that small accretion disc diameters are implied; such small bright accretion discs would have occasional strong brightness peaks of several magnitudes that are never observed. This probably tells us that a more complex model is needed, where the time scale of the brightness peaks is related to the crossing time of substructure in the quasar, not the crossing time of the entire quasar. We expect to apply, in a subsequent report, the Schild \& Vakulik (2003) double ring quasar model that successfully models the long history of Q0957+561 microlensing observations.

No systematic multicolor photometric measurements existed until the monitoring program with the NOT was started in 1990 (Østensen et al. 1996). Meanwhile, a suspicion was expressed by Corrigan et al. (1991), and independently by Rix et al. (1992), that the color indices of the components might have changed since the first three-color observations by Yee (1988). This was a very important statement, since reducing different datasets to a single light curve, (e.g. Houde et al. 1994), as well as determining the extinction law in the Q2237+0305 lensing galaxy, (Yee 1988; Nadeau et al. 1991; Falco et al. 1999), are substantially based 
on the assumptions that "all four components have identical intrinsic color indices", and "their observed color differences are due to different degrees of interstellar extinction and reddening by the same extinction law" (Houde et al. 1994), and "the magnification is wavelength-independent... and time-independent", (Falco et al. 1999). In particular, Falco et al. (1999) measured the value of $R_{\mathrm{V}}$ for Q2237+0305 to equal 5.3 and came to a conclusion about great differences in the extinction laws for lensing galaxies from a sample consisting of 23 gravitational lens systems. However, as can be seen from Fig. 4 in Falco et al. (1999), the differences may be significant at wavelengths shorter than $550 \mathrm{~nm}$, while at larger wavelengths the difference between the extinction curves does not exceed the error bars.

In discussing the results of the five-year VRI monitoring of Q2237+0305, Østensen et al. (1996) did not analyze, however, any color changes in the system, having noted only "very nearly equal" colors for components $\mathrm{A}$ and $\mathrm{B}$, as well as roughly equal colors of $\mathrm{C}$ and $\mathrm{D}$, with the extinction difference between the pairs of $0.6^{\mathrm{m}}$ in the $V$ band, provided the extinction law follows $\lambda^{-1}$, according to Houde et al. (1994). Meanwhile, Vakulik et al. (1997) and Burud et al. (1998) reported that the B component became the bluest in the system in 1995, as compared to observations by Yee (1988).

The next step in determining colors and color changes in the Q2237+0305 system was made in VRI observations with the Maidanak 1.5-m telescope in 1997-1998, presented in Dudinov et al. (2000a,b). Variations of colors were argued to be significant, and a tendency of the components to become bluer as their brightness increased was demonstrated with the use of all available multicolor data. Unfortunately, the remarkable monitoring by Wozniak et al. (2000a,b) was done only in the $V$ band, and thus can not be used to investigate the color changes, while the most recent data of the GLITP collaboration have been taken in $V$ and $R$ filters for a campaign of 4 months only (Alcalde et al. 2002).

By this time, a great amount of observations of Q2237+0305 in spectral ranges other than visual continuum exists - VLA observations at $20 \mathrm{~cm}$ and $3.6 \mathrm{~cm}$ (Falco et al. 1996), observations in the near and mid-IR (Nadeau et al. 1991; Agol et al. 2000), and in the quasar emission lines (Fitte \& Adam 1994; Racine 1992; De Robertis \& Yee 1988; Lewis et al. 1998; Saust 1994). The observed magnitudes of the components have been found to be almost unaffected by microlensing in these spectral ranges, which indicates that much larger quasar features radiate in IR and in the radio, as well as in the emission lines, compared to the optical continuum. The recent detection of an arc of C III emission, connecting components A, D and B (Mediavilla et al. 1998), should be regarded as a visual proof of the extended emission line region of the source. Because of the low sensitivity of a large source to microlensing, the brightness ratios for the components, measured in these spectral ranges, were used to test the validity of a great variety of the existing macrolensing models, listed by Wyithe et al. (2002).

Observations in the UV with the HST (Blanton et al. 1998) and the recent X-ray imaging of Q2237+0305 with the Chandra X-ray Observatory (Dai et al. 2003) should also be mentioned here; they provided, in particular, highly accurate relative coordinates of the components (Blanton et al. 1998) and the upper limits for the physical size and brightness of the BroadLine Region producing Ly- $\alpha$ emission, (Dai et al. 2003). Also, the Chandra data permitted the calculation of the time delay between the $\mathrm{A}$ and $\mathrm{B}$ components of $2.7 \mathrm{~h}$.

\section{Observations}

Our observations were carried out with the 1.5-m AZT-22 telescope of the high-altitude Maidanak observatory, (Central Asia, Republic of Uzbekistan), known for its superb seeing conditions and large number of cloudless nights, (Ehgamberdiev et al. 2000). For technical reasons we had to use three different CCD cameras in our observations, a Pictor-416 camera in 1995 , Pictor-416 and TI $800 \times 800$ cameras in 1997 and 1998, and an ST-7 camera in 1999 and 2000. Again for technical reasons, both $f / 8$ and $f / 16$ focal lengths were used in observations. The LN-cooled TI $800 \times 800$ camera, with pixel size of $15 \mu$, kindly provided by Prof. D. Turnshek, unfortunately revealed some peculiarities, caused by the charge transfer inefficiency, which is characteristic for the CCDs of this generation, (Turnshek et al. 1997). In particular, noticeable stretching of stellar images in the direction of charge transfer is seen, as well as a dependence of the PSF upon the coordinates in the chip plane. In addition, sensitivity irregularities of the chip cannot be corrected satisfactorily, as the output of the flat-fielding procedure is dependent on the signal level. All these peculiarities reduced the actual accuracy of the photometry, seen in Tables 4 and 6.

Unfortunately, a poor telescope tracking system sometimes spoiled the intrinsically good seeing of the Maidanak site and did not permit use of exposures longer than $3 \mathrm{~min}$. To provide sufficiently high accuracy of our photometry with such short exposures, we took images in series, consisting of 10 to 20 frames each. The frames were averaged before being subjected to photometric processing, while a comparison of the photometry of individual frames enabled us to obtain an adequate estimate of the random error inherent in a particular series.

Most images have been taken in the $R$ band - 31 dates in 1995-1999, (Table 4), plus 46 dates in 2000, (Table 5) which were obtained almost on a daily basis during 2.5 months. There is also photometry in the $V$ and $I$ bands for 17 dates in 1995-2000 (Table 6). Some results were presented in our previous publications (Vakulik et al. 1997; Bliokh et al. 1999; Dudinov et al. 2000a,b). We present here the results of all our observations, including those which have never been published. In particular, the observations of July-October 2000 are presented, which were undertaken to search for short-period (night-to-night) variations of brightness. The appearance of the Einstein Cross at six epochs between October 1995 and August 2001 can be seen in Fig. 1, which clearly demonstrates high photometric variability in the system.

In addition to the magnitudes of the components, the seeing conditions are also presented in Tables 4 and 5, together with the values of $F W H M$ for particular nights, the scales and the CCD camera used. 


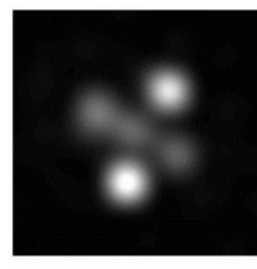

17.09 .1995

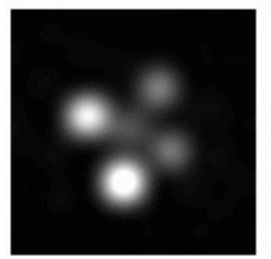

22.07 .1999

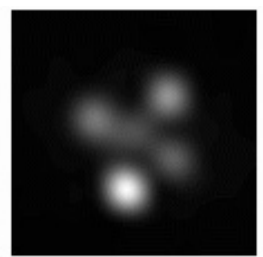

30.08 .1997

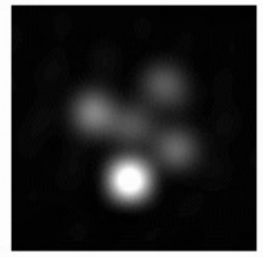

02.08 .2000

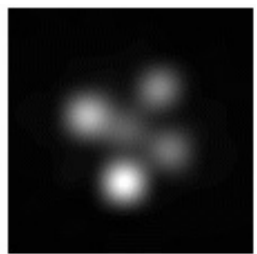

30.08 .1998

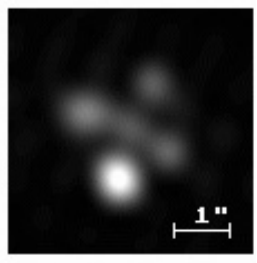

05.08 .2001
Fig. 1. Images of Q2237+0305 for six epochs, obtained in the $R$ band with the $1.5-\mathrm{m}$ Maidanak telescope; component $\mathrm{A}$ is at the bottom, $\mathrm{C}$ is at the left.

\section{Photometric reduction}

The difficulties inherent in accurate photometry of groundbased images of Q2237+0305, have been noted by many authors (Burud et al. 1998; Corrigan et al. 1991; Vakulik et al. 1997; Yee 1988). They are due mainly to its extremely compact spatial structure, with the wings of the quasar images overlapping even under good seeing conditions. Additional difficulties are due to the presence of the rather bright foreground lensing galaxy, with its steep radial brightness distribution. These are the main reasons for the poor agreement of the results of different monitoring programs, and even for a noticeable discrepancy in the photometric results for the same data reduced with different algorithms (Burud et al. 1998; Alcalde et al. 2002). In photometry of the data of 1995-1999, we used the method described in Vakulik et al. (1997), and Bliokh et al. (1999), the general features of which are similar to the double iterative PSF subtraction method, proposed by Yee (1988), who was the first to present spatially resolved photometry of the system. In short, the method is as follows.

The PSF estimate is obtained from a reference star image, and is superimposed upon each image component and the galaxy nucleus successively, and then subtracted in such a way, that no depressions appear in the residual brightness distribution. Such a procedure is repeated iteratively until a stable convergence of estimates of brightness values and coordinates of the components is achieved. Then, according to the resulting estimates, the quasar components are subtracted, and the residual galaxy brightness distribution is smoothed with a rather broad median filter. After the resulting galaxy brightness distribution is subtracted from the initial image, removal of quasar components is repeated, followed again by smoothing the galaxy brightness distribution with a successively decreasing window. The iterative process stops when the width of the median filter becomes of the order of the PSF width.

In processing our data for the 2000 observing season, another method was applied, which used the known relative coordinates of the components and an analytical model of the brightness distribution of the galaxy, represented by the sum of three two-dimensional Gaussian functions. Before describing the algorithm, we will consider the basic principles of photometry for compact groups of star-like objects that have been implemented in the known algorithms of other authors.

Even in the images of Q2237+0305 taken with the Hubble Space Telescope, the quasar components are star-like and thus, in the isoplanatic region, the entire picture (photometric model of the system) can be represented as a sum of the PSFs $r(x-$ $\left.x_{k}, y-y_{k}\right)$, and the galaxy light distribution $g(x, y)$, and in the case of a sampled CCD image, may be written as:

$f(i, j)=\sum_{k=1}^{4} I_{k} r\left(i-x_{k}, j-y_{k}\right)+g(i, j)$,

where $i$ and $j$ are pixel numbers on $x$ and $y$ axes, chosen in parallel to the CCD lines and columns, respectively. The unknown parameters, viz. the coordinates of the components in the detector reference frame, $x_{k}, y_{k}$, their relative brightnesses $I_{k}$, and the galaxy light distribution $g(i, j)$, are usually estimated from a requirement to minimize the difference between the model and the observed brightness distribution in the detected image according to some criterion, e.g. the least-squares criterion:

$\Phi(\boldsymbol{p})=\sum_{i} \sum_{j}(F(i, j)-f(i, j, \boldsymbol{p}))^{2}=\min$.

Here $F(i, j)$ is the brightness distribution in the detected image, and the set of unknown parameters is denoted as $\boldsymbol{p}$ for short. The estimate of the PSF can be obtained from the images of reference stars near the object.

As was noted above, noticeable difficulties in photometry of Q2237+0305 components are caused by the foreground lensing galaxy, as its light distribution $g(x, y)$ is unknown. In minimizing Eq. (2), or another one similar to it, the galaxy brightness distribution is usually represented either analytically (e.g. Burud et al. 1998; Alcalde et al. 2002), or its digital form $g(i, j)$ is estimated, e.g. by using the MCS algorithm (Magain et al. 1998; Burud et al. 1998).

To solve the problem, iterative algorithms are often used, which satisfy Eq. (2), and also make it possible to obtain an estimate of $g(x, y)$ either analytically (Teuber 1993; Ostensen et al. 1996) or in a digital form (Yee 1988; Vakulik et al. 1997). The resulting analytic or numerical model can then be treated as a known function in the photometry of the Q2237+0305 components. Such an approach simplifies the solution procedure, and provides good intrinsic convergence (Corrigan et al. 1991; Alcalde et al. 2002; Burud et al. 1998), but unfortunately, does not ensure the absence of systematic errors in estimating the magnitudes of the components caused by an inadequate galaxy model.

A new image subtraction method proposed by Alard \& Lupton (1999) and successfully applied by Wozniak et al. (2000a,b) and by Alcalde et al. (2002) in Q2237+0305 photometry, seems to be free of this weak point. However, a comparison of photometry results for Q2237+0305 published by the OGLE group and those obtained with other methods reveals some systematic errors in the magnitudes of the components, 
that are probably caused by a bias of brightness estimates in their reference image.

The PSF is usually represented either numerically, or as an analytic function. In this work, the following approach was used. We transformed all the detected images to the same axisymmetrical Gaussian PSF with a preassigned parameter $\sigma_{s}$ using the inverse linear filtration procedure:

$F(i, j)=\tilde{W}\left(\frac{\tilde{F}_{0}\left(\omega_{l}, \omega_{n}\right) \cdot R\left(\omega_{l}, \omega_{n}\right)}{\tilde{r}\left(\omega_{l}, \omega_{n}\right)}\right)$,

here $\tilde{F}_{0}\left(\omega_{l}, \omega_{n}\right)$ is the Fourier transform of the initial image, $F(i, j)$ is the transformed (standardized) image, and $\tilde{W}$ is the inverse Fourier transform operator. A complex-valued inverse filter $w\left(\omega_{l}, \omega_{n}\right)=1 / \tilde{r}\left(\omega_{l}, \omega_{n}\right)$ is composed from the Fourier transform of the initial PSF $r(i, j)$. A function $R\left(\omega_{l}, \omega_{n}\right)=$ $\exp \left[-\sigma_{s}^{2}\left(\omega_{l}^{2}+\omega_{n}^{2}\right) / 2\right]$ forms the Fourier spectrum of the standardized image with the Gaussian PSF for the given parameter $\sigma_{s}$. To construct the inverse filter, a reference star was used about 64" south-west from the quasar, denoted by $\alpha$ in Corrigan et al. (1991).

In doing so, we did not try to increase the resolution in the initial images, and used a transformation (3) that is linear, and, in contrast to non-linear filtration methods, retains photometric accuracy. To exclude dependence of the resulting PSF on the signal-to-noise ratio in the Fourier spectrum of a specific image, we did not use any optimizing algorithms of image reconstruction either, such as e.g. the well-known Wiener filtering.

Since the restoring filter is normalized to unity at zero spatial frequency, such a transformation retains the integral brightness of an image, and thus the estimates of the brightness of the components can be made in units of the brightness of the reference star.

With such standardized images created, the sum in Eq. (1) can be represented as

$s(i, j)=\sum_{k=1}^{4} I_{k} \exp \left\{-\left[\left(i-x_{k}\right)^{2}+\left(j-y_{k}\right)^{2}\right] / 2 \sigma_{s}^{2}\right\}$,

where $\sigma_{s}$ is the effective width of the resulting PSF.

The distribution of light over the galaxy was represented by the sum of three two-dimensional Gaussian functions:

$g(i, j)=\sum_{m=1}^{3} I_{m} \exp \left\{-\left[\left(i-x_{\mathrm{g}}\right) \cos \varphi_{m}+\left(j-y_{\mathrm{g}}\right) \sin \varphi_{m}\right]^{2} / 2 \eta_{m}^{2}\right.$

$$
\left.-\left[-\left(i-x_{\mathrm{g}}\right) \sin \varphi_{m}+\left(j-y_{\mathrm{g}}\right) \cos \varphi_{m}\right]^{2} / 2 \varepsilon_{m}^{2}\right\},
$$

where $x_{\mathrm{g}}, y_{\mathrm{g}}$ are the coordinates of the galaxy center, $I_{m}$ are normalizing coefficients, $\eta_{m}$ and $\varepsilon_{m}$ are parameters determining the characteristic widths of the Gaussian profiles along the major and minor axes respectively, with their meaning understood from Eq. (5), and finally, $\varphi_{m}$ defines the orientations of the major axes. Therefore, the photometric model of the system $f(i, j, \boldsymbol{p})$ in Eq. (2) can be represented as the sum of two constituents, $s(i, j)$ and $g(i, j)$, which describe the quasar components (Eq. (4)), and a photometric model of the light distribution in the lensing galaxy, (Eq. (5)). A set of 26 unknown parameters denoted as $\boldsymbol{p}$ consists of four pairs of
Table 1. Parameters of the photometric model of the lensing galaxy for Q2237+0305 system.

\begin{tabular}{ccccc}
\hline \hline$m$ & $I$ & $\eta\left({ }^{\prime \prime}\right)$ & $\varepsilon\left({ }^{\prime \prime}\right)$ & $\mathrm{PA}\left({ }^{\circ}\right)$ \\
\hline 1 & $0.875 \pm 0.021$ & $0.264 \pm 0.045$ & $0.206 \pm 0.038$ & $57 \pm 3$ \\
2 & $0.090 \pm 0.011$ & $1.260 \pm 0.075$ & $0.790 \pm 0.041$ & $78 \pm 2$ \\
3 & $0.035 \pm 0.002$ & $5.440 \pm 0.530$ & $2.840 \pm 0.110$ & $58 \pm 4$ \\
\hline
\end{tabular}

Table 2. Relative angular positions of Q2237+0305 components A, B, C, D and the galaxy center (G) from observations of 2000 .

\begin{tabular}{ccc}
\hline \hline Component & \multicolumn{1}{c}{$\Delta \alpha\left({ }^{\prime \prime}\right)$} & \multicolumn{1}{c}{$\Delta \delta\left({ }^{\prime \prime}\right)$} \\
\hline $\mathrm{A}$ & 0.000 & 0.000 \\
$\mathrm{~B}$ & $-0.674 \pm 0.003$ & $1.679 \pm 0.004$ \\
$\mathrm{C}$ & $0.624 \pm 0.005$ & $1.206 \pm 0.004$ \\
$\mathrm{D}$ & $-0.867 \pm 0.008$ & $0.513 \pm 0.003$ \\
$\mathrm{G}$ & $-0.085 \pm 0.014$ & $0.939 \pm 0.006$ \\
\hline
\end{tabular}

coordinates $x_{k}, y_{k}$, the coordinates of the galaxy center $x_{\mathrm{g}}, y_{\mathrm{g}}$, normalizing multipliers $I_{k}, I_{m}$, the parameters $\eta_{m}, \varepsilon_{m}$, and the orientations of the axes $\varphi_{m}$ of the three Gaussian components of the galaxy model.

To calculate the parameters of the photometric model of the galaxy, as well as the coordinates of the quasar components, we selected a set consisting of 14 best quality images that was obtained on September 2, 2000 in the $R$ filter under an atmospheric seeing of $0 . ' 8$ and better. The images were averaged and reduced, through the inverse linear filtration procedure described above, to a Gaussian PSF with $\sigma_{s}=0.34$, (FWHM of $0{ }^{\prime} 8$ ).

The least-squares algorithm was used to calculate the brightness values and coordinates of the components and the parameters of the galaxy photometric model from the condition expressed by Eq. (2). Note that the parameters that we obtain for the galaxy model are the result of the convolution of an actual galaxy light distribution with the Gaussian PSF with the given $\sigma_{s}=0{ }^{\prime} 34$. Since Gaussian functions were adopted both for the PSF and for the constituents of the galaxy model, the deconvolved galaxy model parameters can be easily calculated. These deconvolved parameters are presented in Table 1.

In Table 2, the relative positions of the $\mathrm{B}, \mathrm{C}, \mathrm{D}$ components and the galaxy center in the equatorial coordinate system, calculated from the 14 selected images with the procedure described above are presented. Our coordinates agree to 0 .'015 $^{\prime}$ with those obtained from the HST images (Crane et al. 1991; Blanton et al. 1998).

In the subsequent photometric processing of all the available data, every image was reduced to a "standard" PSF, and the corresponding intensities of the quasar images were estimated by minimizing the function (2), with the parameters of the galaxy model and the relative coordinates of the components fixed, according to Tables 1 and 2. The star $\alpha$ from Corrigan et al. (1991) was used as a secondary photometric standard, with its magnitudes taken from their paper. 


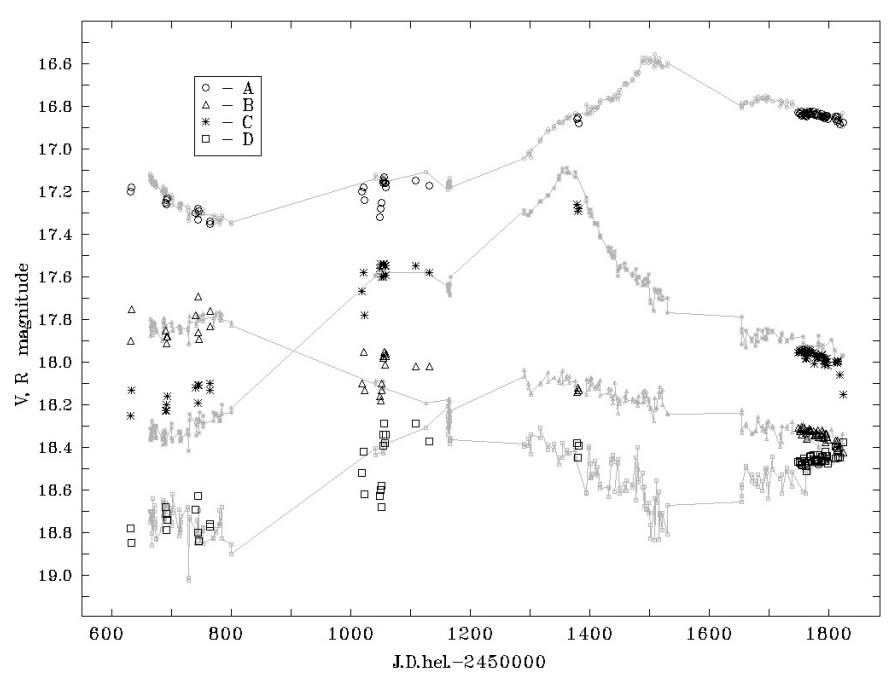

Fig. 2. Photometry of Q2237+0305 A, B, C, D in the $R$ band from observations with the 1.5-m Maidanak telescope in 1997-2000, (large symbols). Photometry in the $V$ band from the OGLE program is also plotted by smaller and fainter symbols. Our data (Tables 4 and 5) are shifted arbitrarily for better comparison, see Sect. 4 for further details. The apparent brightness discrepancy near the 1999 brightness peak of image $\mathrm{C}$ is the result of the use of different filter bands and of a significant color change during the brightness peak.

Photometry of the image sets taken during a single night does not show brightness variations that might be regarded as significant as compared to the photometric errors. Therefore, the brightness estimates taken within a night were averaged, and the formally calculated error in the mean can be regarded as a measure of the inner convergence of our photometry. The method ensures photometry with no seeing-dependent systematic errors, as are inherent in some other methods, for images with a PSF up to 1. .' 4 .

\section{Results of VRI photometry}

Our photometry is presented in Tables 4-6. The zero point for the magnitudes was derived from the reference star from Yee (1988); the magnitudes of the latter have been taken from Corrigan et al. (1991). Our measurements in the $R$ band in 1997-2000 are plotted in Fig. 2, where the OGLE data (Wozniak et al. 2000b) taken in the $V$ filter are shown in grey. For a better comparison, our data of Tables 4 and 5 are shifted by small amounts along the vertical axis, viz. $0.1,0.13,0.15$ and 0.3 mag for $\mathrm{A}, \mathrm{B}, \mathrm{C}$ and $\mathrm{D}$, respectively.

The most important brightness changes observed were:

1. An increase of the brightness of image A, starting at the end of 1998 and peaking, according to the photometry of Wozniak et al. (2000b) and Alcalde et al. (2002), in the middle of November 1999. We observed almost $0.4^{\mathrm{m}}$ brightening of image A between our observing seasons in 1998 and 1999.

2. A monotonic decline of almost $1.0^{\mathrm{m}}$ in the brightness of image B starting with our earliest, 1995.8 observation. It had become the faintest component in the $R$ band by September 2000.

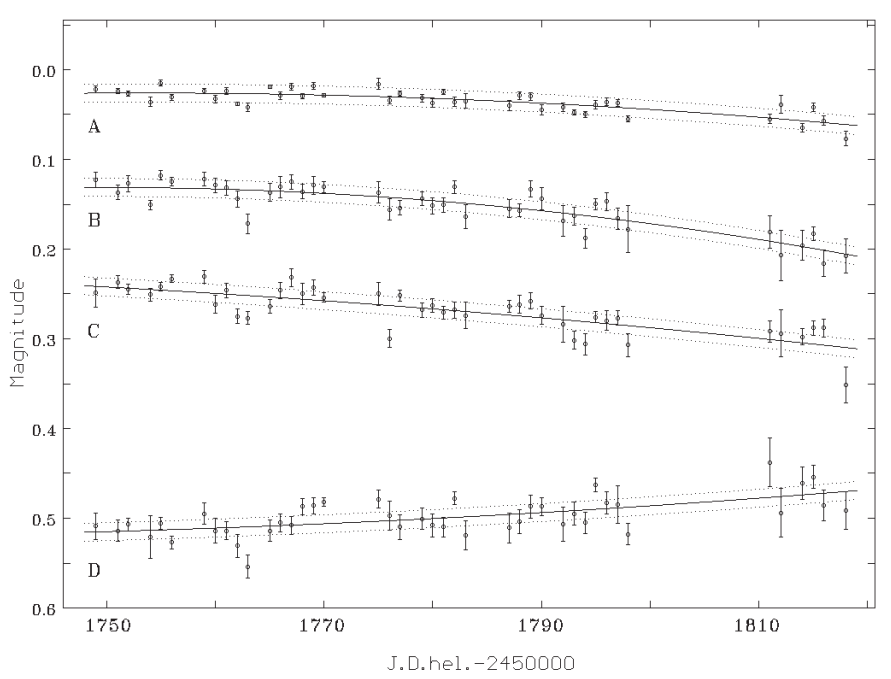

Fig. 3. Photometry of Q2237+0305 A, B, C, D in the $R$ band from observations with the $1.5-\mathrm{m}$ Maidanak telescope in 2000, July 23-September 7 . The light curves of the components are shifted arbitrarily along the magnitude axis with respect to each other for clarity, and approximated by second-order polynomials.

3. A strong brightness peak in image C. Our observations in July 19-22, 1999 were made near the brightness peak of the $\mathrm{C}$ component seen in the well-sampled light curves of Wozniak et al. (2000b). The C image became almost $1^{\mathrm{m}}$ brighter in the $R$ band between August 1997 and July 1999. Thus we have an excellent occasion to detect the color change that accompanied the brightness peak.

4. A noticeable increase of the brightness of image D, which is no longer the faintest one since September 2000.

Our measurements presented in Fig. 2 are in good qualitative agreement with more detailed and accurate single-filter light curves of Wozniak et al. (2000b), taken in the $V$ band for a similar epoch. The large scatter of points for 1997 and 1998 in Fig. 2 is due to the dates when the TI $800 \times 800$ CCD camera was used. We compared our $V$ magnitudes, taken with the TI $800 \times 800$ camera in 1997-98, (Table 6) with the same dates of OGLE monitoring, and found that the OGLE $V$ magnitudes are systematically smaller than our measurements with this camera. The greatest differences are for the A and D components, reaching approximately $0.2^{\mathrm{m}}$, with $0.1^{\mathrm{m}}$ for B and C. As seen from comparison of Table 4 with Table 5, where the photometry with the ST-7 camera is presented, the latter is almost an order of magnitude more accurate than the TI $800 \times 800$ data.

Our best-sampled and most accurate measurements, made in July-September 2000 with the ST-7 camera - the datapoints near the right edge of Fig. 2 - can be seen in Fig. 3 more in detail, (see also Table 5). For clarity, the light curves in Fig. 3 were arbitrarily shifted along the magnitude axis, and fit with quadratic polynomials, with the $1 \%$ error strips shown. The brightness variations of all the components were moderate during this time period, about $0.02^{\mathrm{m}} \div 0.03^{\mathrm{m}}$ per month, and are approximated quite well by the second-order polynomials. The brightness estimates for the A component are mainly within a $1 \%$ deviation with respect to the fitted curve. A correlation between the rapid brightness variations of all the components 
Table 3. A comparison of three programs of Q2237+0305 photometry: Maidanak (this work, ST-7 CCD), OGLE and GLITP; $V$ magnitude differences for A, B, C and D components; observations of 2000.

\begin{tabular}{ccccc}
\hline \hline Programs & $\Delta V_{\mathrm{A}}$ & $\Delta V_{\mathrm{B}}$ & $\Delta V_{\mathrm{C}}$ & $\Delta V_{\mathrm{D}}$ \\
\hline GLITP-OGLE & 0.07 & 0.01 & 0.14 & -0.18 \\
Maidanak-OGLE & 0.06 & 0.05 & 0.07 & -0.15 \\
\hline
\end{tabular}

seen in Fig. 3 could be ascribed to quasar-intrinsic brightness changes, except that since their amplitudes are larger for the fainter components, these variations are probably not real and are more likely due to errors.

We compared our photometry of July-September 2000 in the $V$ band with the OGLE data, obtained for the same dates, and, since our data do not overlap with the observations of the GLITP collaboration, we made a similar comparison between their photometry and that of OGLE. The results of this comparison are presented in Table 3. Here, positive differences mean that the OGLE magnitudes are smaller. The difference between our photometry and that of the OGLE program will be even smaller for A, B and C images if one takes into account the $0.034^{\mathrm{m}}$ difference in magnitude for star $\alpha$ adopted in Wozniak et al. (2000a) and in this work, though the systematicerrors for the $\mathrm{C}$ component will become larger.

\section{Variations of color in Q2237+0305}

The first multicolor observations by Yee (1988) showed that the components have different colors. An obvious dependence of the components' reddening on the distance to the galaxy nucleus was explained by Yee as selective extinction in the dusty matter of the lensing galaxy. This made it possible to estimate the extinction law in the lensing galaxy, which, according to Nadeau et al. (1991) and Yee (1988), is similar to that in our Galaxy. It should be emphasized here, that the conclusion was based on the analysis of color differences of the components for a fixed epoch.

As mentioned in the Introduction, a suspicion arose in 1991 and 1992 that the colors of the components might have changed, (Corrigan et al. 1991; Rix et al. 1992). In particular, Corrigan et al. did not find any significant variations of $(B-r)$ colors of the components with time, but they were the first to notice that "there may be a small color change in image A as the $r$ magnitude gets fainter" (Corrigan et al. 1991). They referred to the work by Wambsganss \& Paczinski (1991), where the possibility is discussed that, if the quasar structure is wavelength-dependent, microlensing events will differently reveal themselves in different spectral regions. In particular, according to Wambsganss \& Paczinski (1991), the bluer inner parts of the continuum source might be more strongly amplified than the outer parts.

Rix et al. (1992), analyzing their observations in the $U$ and $R$ bands with the Hubble Space Telescope, plotted their $(U-R)$ colors against the $(g-i)$ colors of the components measured by Yee (1988), and concluded that they are "only marginally consistent" with the reddening line derived by Nadeau et al. (1991). They suggested that the discrepancy could be due to either variable dust extinction in the lensing galaxy, or to the effects of microlensing color changes, first noted by Kayser et al. (1989) and later investigated by Wambsganss \& Paczynski (1991) and Wambsganss (1991) in simulations.

We have already analyzed the behavior of the relative colors of the Q2237+0305 components qualitatively (Dudinov et al. 2000a,b), based upon our observations at Maidanak in 1995 (Vakulik et al. 1997), and in 1997-1998, and also upon all available multicolor observations by other authors (Burud et al. 1998; Østensen et al. 1996; Rix et al. 1992; Yee 1988). A tendency for the components to become bluer as their brightness increases was noted there, but no quantitative relationships were derived.

We present here our measurements of the colors of the A, B, C, D components, and the attempt to quantitatively analyze the behavior of the $(V-R)$ and $(V-I)$ color indices of the components using our data taken in 1995-2000. VRI photometry is presented in Table 6 , and $(V-R)$ and $(V-I)$ colors are listed in Table 7. Formal errors for these quantities, calculated as the errors of the average, range from $0.02^{\mathrm{m}}-0.03^{\mathrm{m}}$ (component A) to $0.03^{\mathrm{m}}-0.05^{\mathrm{m}}$ (component $\mathrm{D}$ ), for the most accurate observations of 2000, and, as seen from Table 6, are within $0.08^{\mathrm{m}}-0.15^{\mathrm{m}}$ for the observations of $1997-1998$ made with the TI $800 \times 800$ camera.

As shown in the previous section, our photometry is in quite satisfactory agreement with that obtained by other observers for nearby epochs, (e.g. Alcalde et al. 2002; Woznyak et al. $2000 \mathrm{~b})$. At any rate, the discrepancy does not exceed that obtained when different algorithms are applied to the same data, (Alcalde et al. 2002; Burud et al. 1998).

However, one should keep in mind the peculiarities of the Q2237+0305 photometry mentioned above in analyzing and interpreting the lightcurves in general, and especially those combined from heterogeneous observing data. With this in view, more weight should be given to the analysis of relative quantities, which are less sensitive to differences in observational circumstances and image processing algorithms. Such quantities are relative colors and relative magnitudes, as well as their variations. Examining their behavior in time, and their relationships with each other in microlensings can be a valuable source of additional information about the physical properties of both the quasar and lensing galaxy. In particular, they can be used to probe the spatial structure of the quasar at different wavelengths (Wambsganss \& Paczynski 1991), and to determine the extinction law in the lensing galaxy.

A correlation between the $(V-I)$ colors of the components and their $R$ magnitudes can be seen in Fig. 4, where the components are marked with different symbols. Note that components $\mathrm{B}, \mathrm{C}$ and $\mathrm{D}$ lie along a line in this diagram, while the component A forms a separate cluster of points. We cannot refute the possibility of some systematic error in our photometry, but such an error would hardly cause components B, C and D to lie on a single line (the correlation coefficient is 0.8 ) while separating out the A component. Moreover, we studied the systematic errors of our algorithms very carefully in simulation and found that their effect, if present, would cause only a slight bias in the color of the $\mathrm{C}$ and $\mathrm{D}$ components to larger values, 


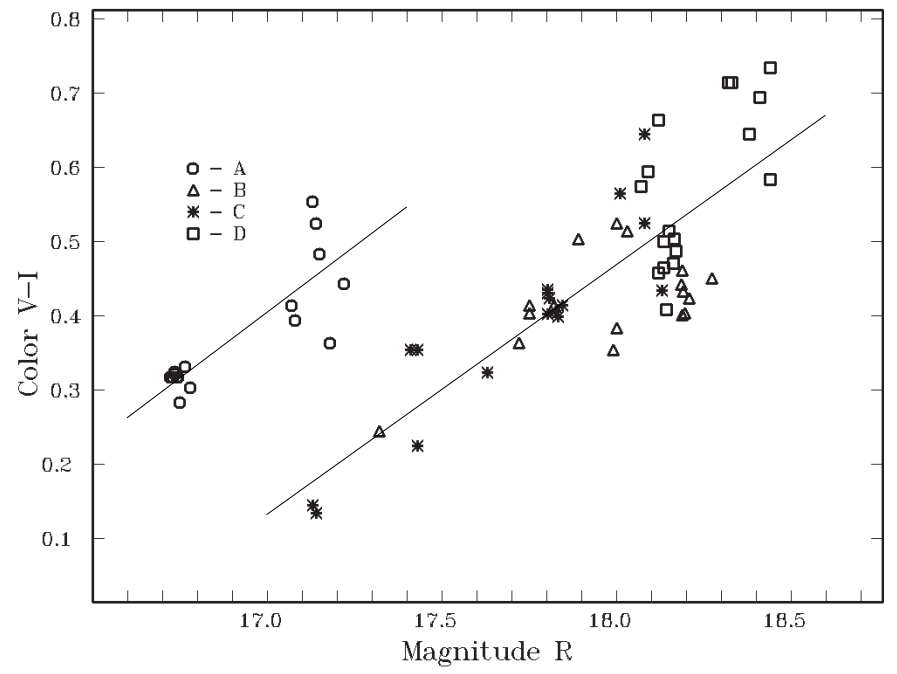

Fig. 4. $(V-I)$ colors vs. $R$ magnitudes calculated for the observations of 1995-2000. Note the separate cluster of points for component $\mathrm{A}$. The slope of the regression line for components $\mathrm{B}, \mathrm{C}$ and $\mathrm{D}$ is $0.33 \pm 0.08$.

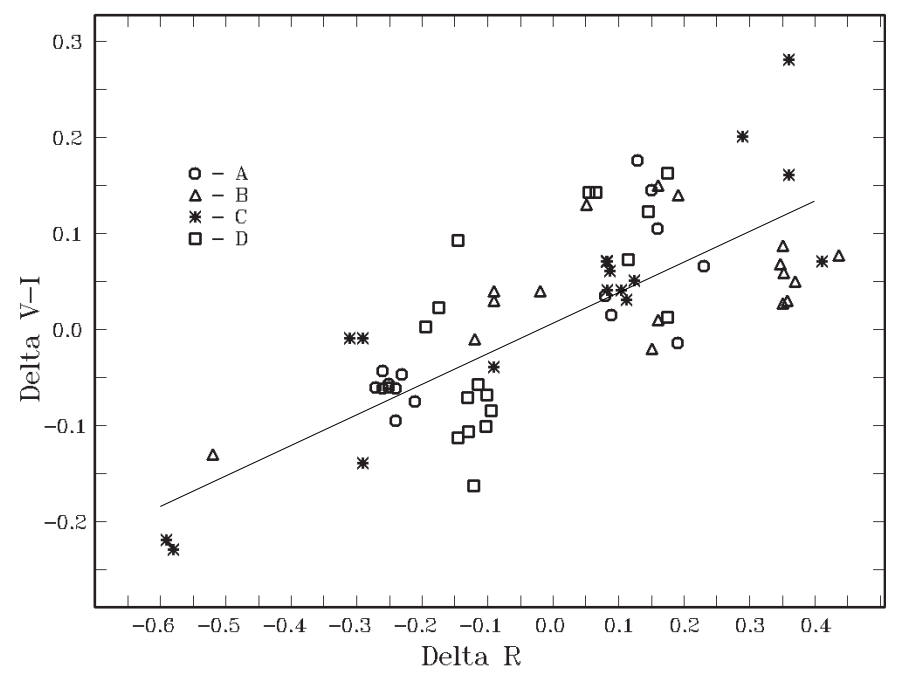

Fig. 5. Variations of $(V-I)$ colors (vertical axis) vs. variations of $R$ magnitudes, calculated for observations of 1995-2000. The slope of the regression line is $0.31 \pm 0.08$.

i.e. make them redder than A and B (see Vakulik et al. 1997 for more details). We see from Fig. 4, however, that the cluster of points for component $\mathrm{A}$ is shifted towards redder colors with respect to the points for $\mathrm{B}, \mathrm{C}$ and $\mathrm{D}$.

If all the components were equally amplified, and if both the colour differences of the components and their variations in time were caused by an interstellar reddening law similar to that for our Galaxy, a linear relationship between $(V-I)$ and $R$ could be expected, with a regression slope of about 0.42 for $(V-I)$ (Schild 1977). Microlensing events, with their still unknown brightness-color dependence, would disturb and rearrange this order, making the components follow the reddening line on average, but forming individual clusters of points, with the patterns and stretches determined by the level of microlensing activity at a particular period of time, and by the unknown character of the color-brightness dependence of microlensing.
However, the existing macrolens models predict different macroamplifications for the components. According to the macromodel by Schmidt et al. (1998), rather well confirmed by the observations in emission lines (Fitte \& Adam 1994; Racine 1992; Lewis et al. 1998; Saust 1994), and in the IR spectral range (Nadeau et al. 1991; Agol et al. 2000), where no microlensing effects are expected, the A, B and D components must be almost equally amplified, with flux ratios of $0.25,0.27$ and 0.32 respectively. The $\mathrm{C}$ component is expected to have the lowest macroamplification factor by this model, a flux ratio of 0.15 is predicted for it. This means that in this case the components cannot be expected to lie along a line in the $(V-I)$ vrs $R$ plot. In the presence of microlensing, the components would produce a family of clusters, shifted with respect to each other by the amount of flux ratio differences.

However, we see in Fig. 4 another situation. While components $\mathrm{B}, \mathrm{C}$ and D produce three overlapping clusters, all of them stretched approximately in the same direction, the A datapoints form a separate cluster, stretched along a line with a slope similar to that of the joint B, C and D cluster. All the points in the joint cluster are rather well correlated, with a correlation index of $0.8 \pm 0.1$ and a regression line slope of $0.33 \pm 0.08$. The points formed by the A component are also rather well correlated, with a correlation index of 0.84 and a regression line slope of 0.36 .

To eliminate possible additive constituents of the colormagnitude dependence, which may differ for different components, and to focus on the analysis of changes, we studied a correlation between the deviations of the $(V-I)$ colors from their average over the whole time period, and the brightness variations in the $R$ band. The diagram can be seen in Fig. 5 . The quantities are rather well correlated, with a correlation index of $0.75 \pm 0.08$, and a regression slope of $0.31 \pm 0.08$, in good agreement with that of Fig. 4 for components B, C and D, but the A component is not separate here. The uncertainties are given for an $80 \%$ confidence interval.

A diagram of $(V-I)$ vs. $(V-R)$, which is known to be of great diagnostic importance for the study of dust extinction, is usually presented in all papers on multicolor observations, e.g., Yee (1988), Rix et al. (1992), Burud et al. (1998), but, as was noted above, for fixed epochs. In such a diagram, the color indices should be proportional to each other for any color base and for any type of dust extinction, with the slope determined by the reddening law. The diagram built with the use of our measurements (see Table 6), is shown in Fig. 6. A large range of color variations of the $\mathrm{C}$ component should be particularly noted. It is quite real and can be explained by the presence of observations of 1999 in our data, viz. two asterisks near the origin. As was noted, these data were obtained near the July 1999 brightness peak of $\mathrm{C}$, when it became almost $1^{\mathrm{m}}$ brighter during two years (Wozniak et al. 2000b), and exceeded the B component in brightness. The datapoints in this diagram are found along a line with a slope of approximately $1.31 \pm 0.14$, which is much less than the slope of 2.16 for these color indices expected for the interstellar reddening law in our Galaxy (Schild 1977). We conclude that if the extinction law in the lens galaxy is similar to that of our Galaxy, the observed color changes cannot be explained by variable interstellar reddening. 


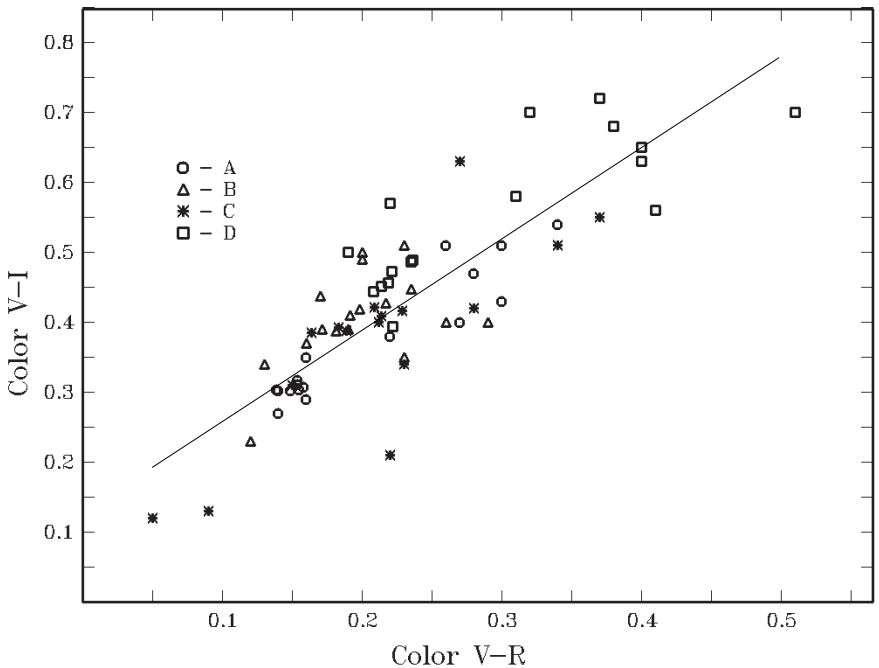

Fig. 6. $(V-I)$ vs. $(V-R)$ diagram for $\mathrm{Q} 2237+0305$ components A, B, C, D plotted from the Maidanak data, taken in 1995-2000. The slope of the regression line is $1.31 \pm 0.14$.

\section{Color changes associated with brightness peaks}

A further perspective of the nature of the observed color changes comes from a comparison of the history of brightness changes with the history of color changes. The general features of long-term variations of the component magnitudes during 1995-2000 in comparison with the simultaneously determined colors can be seen in Fig. 7. Since the durations of our observing sessions are short compared to the characteristic time scale of the long-term variations of colors of the components, we calculated the mean values for $(V-I)$ and $R$ for every session, and plotted them as a function of time (the midpoint dates of each session are used here).

Long-term variations of the colors of the components are clearly seen in this figure, as well as a tendency of the components to shift their color indices towards smaller values (bluer color) as their brightness increases. But the tendency is not always straightforward. Some reddening of the components, preceding the subsequent decrease of their color indices at the stage of component brightening can be also seen. As noted above, our observations in July 1999 were made very close to the brightness peak of component $\mathrm{C}$, which is seen very well from the more complete and well sampled light curves of the OGLE program (Wozniak et al. 2000b), while the A component was just in the middle of the ascending slope of its peak at this time, according to the observations of OGLE and GLITP programs (Wozniak et al. 2000b; Alcalde et al. 2002).

The relationship between brightness change and color is obvious and approximately as expected from models of Wambsganss \& Paczynski (1991). The most direct correlation is found for image $\mathrm{C}$, where $(V-I)$ is almost perfectly anticorrelated with brightness. Thus as the quasar image $\mathrm{C}$ brightened by almost $1.0^{\mathrm{m}}$ in $R$ between 1997.5 and 1999.6, it became bluer by 0.42 magnitudes in $(V-I)$ (Fig. 7). A second interesting type of behavior is seen in the brightness of image A, where we found to increase by $0.45^{\mathrm{m}}$ as $(V-I)$ became bluer

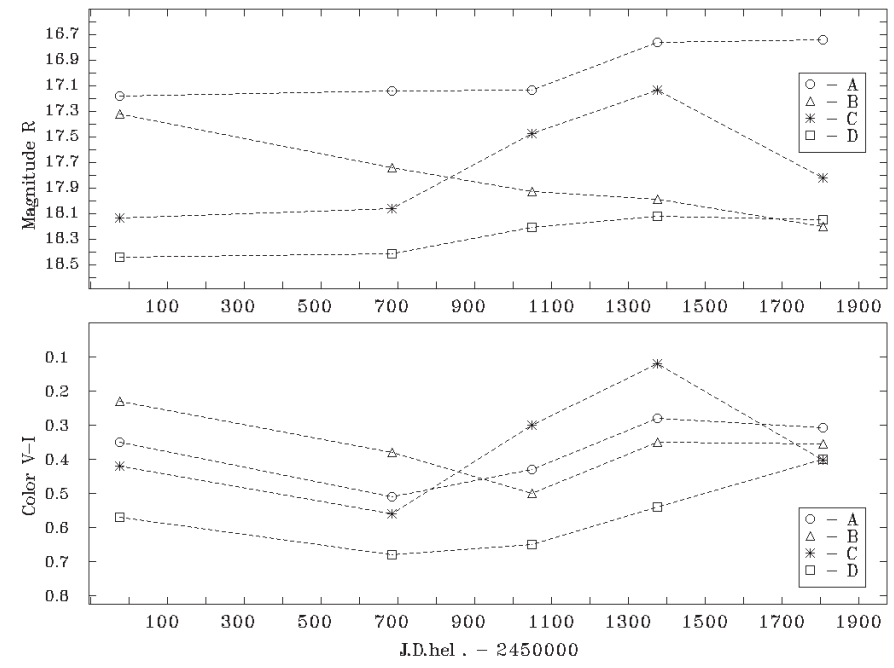

Fig. 7. Long-term variations of $R$ magnitudes (upper panel) and ( $V-$ I) colors (at the bottom) of components A, B, C, D of Q2237+0305 from the observations of 1995-2000. Each point is a result of averaging within one observational set.

by $0.15^{\mathrm{m}}$. Just as interesting is the color history for image B, which underwent a sustained slow brightness drop of $1.0^{\mathrm{m}}$ during our monitoring period. As it slowly declined in brightness, it became $0.25^{\mathrm{m}}$ redder in $(V-I)$ from 1995.7 to 1998.8 , and then again became $10 \%\left(0.1^{\mathrm{m}}\right)$ bluer as the brightness continued to fade from 1998.8 to 2000.9 .

We have already shown that this is not likely to be produced by a hole appearing in some absorbing clouds. If instead we view the image $C$ brightness peak as a microlensing artefact where a compact object (star) in the lens galaxy passes in front of the quasar and causes the temporary brightening, we can make a comparison with the calculations in Figs. 1-3 of Wambsganss \& Paczinski (1991). Their models were crafted to apply to Q2237, and they show approximately the correct brightness change $\left(1^{\mathrm{m}}\right.$ increase in $\left.V\right)$ and color change $\left(0.4^{\mathrm{m}}\right.$ bluer in $\left.(B-R)\right)$ for events with 1 year duration, and appear similar to the Wambsganss \& Paczinski (1991) Fig. $2 i, j$ pattern of a quasar image passing outside the cusp of a microlensing star. We do not continue these calculations because we feel that the failed prediction of Wyithe et al. (2000c) of a subsequent large brightness change invalidates all models with such simple accretion disc approximations. However, almost any quasar model with an energizing central source produces quasar structure which is more compact at shorter wavelengths. We expect to produce separately a series of models that can reproduce the observed effects, based upon the double-ring Schild \& Vakulik (2003) model.

Although quasar emission lines contaminate the color photometry in the continuum-dominated filter bands, we doubt whether the emission lines are responsible for the large brightness-color effects found here, given that the large brightness changes observed are always associated with microlensing of the quasar continuum. 


\section{Conclusions}

1. Our observations show drastic changes of the component magnitudes, which are inherently uncorrelated in this system, confirming the high probability for microlensings, predicted for Q2237+0305 in 1989 (Kayser \& Refsdal 1989). The highest gradient of brightness change was observed for the C component between 1997 and 1998 - almost $0.07^{\mathrm{m}}$ per month in our $R$ filter. Almost the same value was measured by the OGLE program in their $V$ band for the same time period. However, a much more rapid brightness change of the $\mathrm{C}$ component was detected immediately after its extraordinary brightness peak in July 1999 by the OGLE program - almost a $0.2^{\mathrm{m}}$ decrease per month in their $V$ band (Wozniak et al. 2000b).

2. No strong microlensing event occurred in the system during our detailed 2.5-months monitoring in July-October 2000 apart from the fact that the $\mathrm{B}$ component has become the faintest one, after its long continuous fading beginning in 1995 (see Fig. 2 and Table 5). No noticeable nightto-night brightness variations were detected during this time period. Moderate brightness changes were inherent in all the components, reaching a $0.03^{\mathrm{m}}$ decrease per month for B and C, and $0.02^{\mathrm{m}}$ brightening for D (see Fig. 3 and Table 5).

3. All the components showed variations of their colors during 1995-2000, which we argue to be real and significant. The most prominent change of color was observed for the C component, $-0.43^{\mathrm{m}}$ for its $(V-I)$ color index during two years. The $(V-I)$ color indices of A, B and D were less variable during the whole time period, changing from $0.3^{\mathrm{m}}$ to $0.5^{\mathrm{m}}$ for $\mathrm{A}$, from $0.2^{\mathrm{m}}$ to $0.5^{\mathrm{m}}$ for $\mathrm{B}$, and from $0.7^{\mathrm{m}}$ to $0.45^{\mathrm{m}}$ for $\mathrm{D}$ component, which became $0.2^{\mathrm{m}}$ bluer between 1997 and 2000, having approached the B component in color and exceeded it in brightness.

4. The $(V-I),(V-R)$ color-color plot shown in Fig. 6 incorporates all our observations. The regression slope is $1.31 \pm 0.14$ for this diagram, i.e. much smaller than the value of 2.16 expected from the reddening line in our Galaxy for these color indices. We conclude that the changes in brightness and color are not caused by time variations in reddening, but are more probably caused by microlensing of source structure that is more compact at shorter wavelengths.

5. The large brightness peak of the C component in July 1999 was accompanied by large color change in the sense that as the $\mathrm{C}$ image brightness increased by almost $1^{\mathrm{m}}$ both in our $R$ and in the Wozniak et al. (2000b) $V$ bands, the color became bluer by $0.43^{\mathrm{m}}$ in $(V-I)$. This is the sense and amplitude expected for microlensing of an object that is smaller at shorter wavelengths, as modelled previously by Wambsganss \& Paczinski (1991). The colors of the other components behave similarly, though the amplitudes of their color variations are smaller (see Fig. 7).

6. Returning to Fig. 4, where the relationship between the $(V-I)$ colors and $R$ magnitudes of the components is shown, we note that the plot is inconsistent with the adopted models of macrolensing, e.g. Schmidt et al. (1998).
We think that most probably the A component is macroamplified almost $0.8^{\mathrm{m}}$ more than $\mathrm{B}, \mathrm{C}$ and $\mathrm{D}$, which have almost equal amplifications.

We hope that the data presented here will demonstrate the importance of multiband observations of gravitationally lensed quasars in general, and Q2237+0305 in particular. A more detailed analysis of the obtained data and simulation with the new quasar structure model, will be presented in our next paper, which is in progress.

Acknowledgements. The authors thank the Maidanak Foundation, and its President Dr. Henrik N. Omma personally for delivering the ST-7 CCD camera. We also appreciate valuable financial support and kind attention to our work from Dr. James Bush and Prof. Kim Morla (Pontificia Universidad Catolica del Peru, Lima). The work has also been substantially supported by the joint UkrainianUzbek Program "Development of observational base for optical astronomy on Maidanak Mountain". The observations of 1997-98 have become possible thanks to funding from the CRDF grant UP2-302, with Prof. B. Paczynski as a US Co-Investigator, whom the authors from Ukraine greatly appreciate. The co-authors from Russia are also thankful to the Russian Foundation of Fundamental Research, grants No. 98-02-17490 and 1.2.5.5.

\section{References}

Agol, E., \& Krolik, J. 1999, ApJ, 524, 49

Agol, E., Jones, B., \& Blaes, O. 2000, ApJ, 545, 657

Alard, C., \& Lupton, R. H. 1998, ApJ, 503, 325

Alcalde, D., Mediavilla, E., Moreau, O., et al. 2002, ApJ, 572, 729A

Blanton, M., Turner, E. L., \& Wambsganss, J. 1998, MNRAS, 298, 1223

Bliokh, P. V., Dudinov, V. N., Vakulik, V. G., et al. 1999, Kin. Phys. Cel. Bodies, 15, 338

Burud, I., Stabell, R., Magain, P., et al. 1998, A\&A, 339, 701

Corrigan, R. T., Irwin, M. J., Arnaud, J., et al. 1991, AJ, 102, 34

Crane, P., Albrecht, R., Barbieri, C., et al. 1991, ApJ, 102, 34

Cumming, C. M., \& De Robertis, M. M. 1995, PASP, 107, 469

Dai, X., Chartas, G., Agol, E., et al. 2003, ApJ, 589, Iss. 1, 100

De Robertis, M. M., \& Yee, H. K. C. 1988, ApJ, 332, L49

Dudinov, V. N., Vakulik, V. G., Zheleznyak, A. P., et al. 2000a, Kin. Phys. Cel. Bodies, 16, 346

Dudinov, V., Bliokh, P., Paczynski, P., et al. 2000b, Kin. Phys. Cel. Bodies Suppl. No. 3, 170

Ehgamberdiev, S. A., Baijuramov, A. K., Ilyasov, S. P., et al. 2000, A\&AS, 145, 293

Falco, E. E., Lehar, J., Perley, R. A., et al. 1996, AJ, 112, 897

Falco, E. E., Impey, C. D., Kochanek, C. S., et al. 1999, ApJ, 523, 617

Fitte, C., \& Adam, G. 1994, A\&A, 282, 11

Houde, M., \& Racine 1994, AJ, 107, 466

Irwin, M. J., Webster, R. L., Hewett, P. C., et al. 1989, AJ, 98, 1989

Kayser, R., Refsdal, S., \& Stabell, R. 1986, A\&A, 166, 36

Kayser, R., \& Refsdal, S. 1989, Nature, 338, 745

Lewis, G. F., \& Irwin, M. J. 1996, MNRAS, 283, 225

Lewis, G. F., Irwin, M. J., Hewett, P. C., \& Foltz, C. B. 1998, MNRAS, 295,573

Magain, P., Courbin, F., \& Sohy, S. 1998, ApJ, 494, 472

Mediavilla, E., Arribas, S., del Burgo, C., et al. 1998, ApJ, 503, L27

Nadeau, D., Yee, H. K. C., Forrest, W. J., et al. 1991, ApJ, 376, 430

Østensen, R., Refsdal, S., Stabell, R., et al. 1996, A\&A, 309, 59

Refsdal, S., \& Stabell, R. 1993, A\&A, 278, L5

Racine, R. 1991, AJ, 102, 454 
Racine, R. 1992, ApJ, 395, L65

Rix, H.-W., Schneider, D. P., \& Bachcall, J. N. 1992, AJ, 104, 959

Saust, A. B. 1994, A\&AS, 103, 33

Schild, R. 1977, AJ, 82, 337

Schild, R., \& Vakulik, V. 2003, AJ, 126, 689

Schmidt, R., Webster, R. L., \& Lewis, G. F. 1998, MNRAS, 295, 488

Schmidt, R. W., Kundic, N., Pen, U.-L., et al. 2002, A\&A, 392, 773

Teuber, J. 1993, Digital image Processing, Prentice-Hall

Turnshek, D. A., Lupie, O. L., Rao, S. M., et al. 1997, ApJ, 485, 100

Vakulik, V. G., Dudinov, V. N., Zheleznyak, A. P., et al. 1997, Astron. Nachr., 318, 73

Wambsganss, J., Paczynski, B., \& Schneider, P. 1990, ApJ, 358, L33

Wambsganss, J. 1992, Gravitational lenses, Lect. Notes Phys., 406, 183

Wambsganss, J., \& Paczinski, B. 1991, AJ, 102, 864
Webster, R. L., Ferguson, A. M. N., Corrigan, R. T., \& Irwin, M. J. 1991, AJ, 102, 1939

Wozniak, P. R., Alard, C., Udalski, A., et al. 2000a, ApJ, 529, 88

Wozniak, P. R., Udalski, A., Szymanski, M., et al. 2000b, ApJ, 540, L65

Wyithe, J. S. B., Webster, R. L., \& Turner, E. L. 2000a, MNRAS, 318, 762

Wyithe, J. S. B., Webster, R. L., \& Turner, E. L. 2000b, MNRAS, 312, 843

Wyithe, J. S. B., Turner, E. L., \& Webster, R. L. 2000c, MNRAS, 318, 1105

Wyithe, J. S. B., Agol, E., \& Fluke, C. J. 2002, MNRAS, 331, 1041

Yee, H. K. C. 1988, AJ, 95, 1331

Yonehara, A., Mineshige, S., Manmoto, T., et al. 1998, ApJ, 501, L41

Yonehara, A. 2000, ApJ, 548, L127 